\title{
EFEITOS HEMODINÂMICOS DA INTOXICAÇÃO AGUDA COM BUPIVACAÍNA E A MISTURA ENANTIOMÉRICA - ESTUDO EXPERIMENTAL EM SUÍNOS
}

\author{
Artur Udelsmann*, Sillvia Elaine Rodolfo de Sá lorena, Samira Ubaid Girioli, William Adalberto Silva, Ana Cristina de Moraes
}

Trabalho realizado no Laboratório de Anestesia Experimental do Núcleo de Medicina e Cirurgia Experimental da FCM / UNICAMPm, Campinas, SP

\section{*Correspondência}

Av. Prof. Atílio Martini, 213

Campinas - SP

Cep 13083-830

Tel.: (19) 3289-4651 / 9105-8304

Fax: (19) 3521-9561

audelsmann@yahoo.com.br

\begin{abstract}
RESUMO
OBjetivos. A bupivacaína racêmica tem sido o anestésico local de escolha nos bloqueios regionais pela qualidade e duração de sua anestesia. Sua cardiotoxicidade é motivo de preocupações, e pesquisas têm sido realizadas para encontrar drogas com menor impacto. Seu isômero levógiro, a levobupivacaína, seria menos cardiotóxico pela menor afinidade aos receptores dos canais de sódio do coração, e é uma opção. Em nosso país, uma apresentação contendo 75\% do isômero levógiro e 25\% do isômero dextrógiro, denominada mistura enantiomérica, está disponível. O objetivo deste estudo foi comparar as repercussões hemodinâmicas da injeção intravascular de uma dose tóxica destes dois agentes em suínos.

Métodos. Súnos da raça Large White foram anestesiados com tiopental, entubados e ventilados mecanicamente, sendo, em seguida, instalada monitorização hemodinâmica com pressão invasiva e cateter de Swan-Ganz numa artéria pulmonar. Após repouso de 30 minutos, os animais foram divididos aleatoriamente em dois grupos, e foi realizada em duplo-cego intoxicação com um dos agentes na dose de $4 \mathrm{mg} / \mathrm{kg}$. Os resultados hemodinâmicos foram avaliados então a um minuto, aos cinco, 10, 15, 20 e 30 minutos.
\end{abstract}

REsultados. A mistura enantiomérica causou maiores repercussões hemodinâmicas do que a bupivacaína racêmica. Estes resultados se opõem aos encontrados em humanos com o isômero levógiro, mas estão de acordo com achados recentes em animais. Extrapolar resultados de animais para seres humanos requer cautela, e novas pesquisas são necessárias.

Conclusão. Em suínos, a mistura enantiomérica mostrou-se mais tóxica do que a bupivacaína racêmica, quando grandes doses são injetadas por via endovenosa.

Unitermos: Anestésicos. Bupivacaína. Mistura enantiomérica. Hemodinâmica. Intoxicação.

\section{INTRODUÇÃO}

Anestesias loco-regionais requerem, às vezes, doses elevadas de anestésicos locais, e sempre há o risco de ocorrerem reações tóxicas, sobretudo nos sistemas cárdio-vascular e nervoso central, em caso de injeções inadvertidas intravasculares. A bupivacaína, em razão da qualidade de sua anestesia e sua ação prolongada, é um dos anestésicos locais mais utilizados atualmente ${ }^{1,2}$. Entretanto, desde a publicação, em 1979, de editorial na revista Anesthesiology sobre graves efeitos cárdiovasculares da intoxicação com esse agente ${ }^{3}$, pesquisas têm sido direcionadas na procura de novos anestésicos locais de longa ação e com menor toxicidade.

Embora a bupivacaína seja sintetizada na forma de seus dois isômeros dextrógiro $R(+)$ e levógiro $L(-)^{4}$, até pouco tempo, ela era comercializada somente na forma de mistura racêmica, contendo $50 \%$ de cada um destes, mesmo que, desde 1972, já fosse evidenciada a menor toxicidade do isômero levógiro 5,6. Recentemente, este isômero levógiro, denominado "levobupivacaína", começou a ser mais estudado e, em modelos animais, foi mostrado que sua dose letal seria até 1,6 maior do que a da mistura racêmica?.

Em humanos, a levobupivacaína teria um menor efeito inotrópico negativo e produziria um menor prolongamento dos intervalos PR e QT do eletrocardiograma, característicos da intoxi- cação da mistura racêmica. Mas este novo agente, embora com a mesma potência analgésica ${ }^{8}$, tem como inconveniente um menor grau de bloqueio motor?

Por esta razão, talvez, a indústria farmacêutica nacional fornece um produto com $75 \%$ de isômero levógiro e $25 \%$ de isômero dextrógiro, conhecido como "mistura enantiomérica". Por tratarse de produto genuinamente nacional, pareceu-nos interessante avaliar qual é o seu impacto hemodinâmico no caso de uma intoxicação acidental, comparando-o aos efeitos da bupivacaína racêmica.

\section{Métodos}

Com objetivo de comparar em suínos os efeitos hemodinâmicos de uma intoxicação aguda com as drogas, 40 suínos da raça Large White, em bom estado de saúde após avaliação veterinária, de ambos os sexos, com peso variando entre 20 e 27 $\mathrm{kg}$, foram submetidos ao seguinte protocolo, após aprovação do Comitê de Ética de Experiências em Animais da UNICAMP:

I. Jejum de véspera com livre acesso à água;

2. Na manhã do protocolo, os animais foram pesados e, após acesso venoso em uma das orelhas, a anestesia foi induzida com tiopental sódico 2,5\% na dose de $25 \mathrm{mg} / \mathrm{kg}^{\prime \prime}$; 


\section{Figura I - Índice cardiáco}

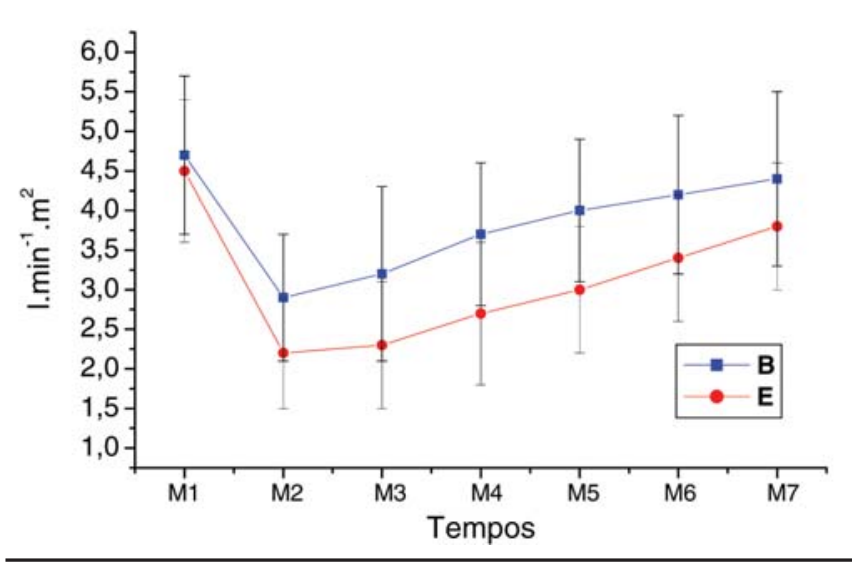

3. Calculou-se a superfície corpórea do animal (SC) em metros quadrados, por meio de fórmula clássica da literatura ${ }^{12}$ : $\mathrm{SC}=(9 \mathrm{x}$ peso em gramas $\left.{ }^{2 / 3}\right) \times 10^{-4}$, introduzindo-a nos parâmetros do monitor multiparamétrico Engstrom AS/3, para cálculo dos valores dos índices corpóreos;

4. Em seguida, o animal foi entubado, conectado a um respirador pneumático por um sistema com reinalaçãa parcial e absorvedor de $\mathrm{CO}_{2}$ com volume corrente de $15 \mathrm{ml} / \mathrm{kg}$, mantendose uma frequêencia respiratória adequada para obter ETCO, entre 32 - $34 \mathrm{mmHg}$. Ao sistema, acrescentou-se fluxo adicional de I litro de $\mathrm{O}_{2}$ e mediu-se a saturação da hemoglobina em oxigênio por meio de sensor colocado na língua do animal, com o objetivo de se ter sempre um valor superior a 97\%. Instalou-se também monitorização ECG;

5. A manutenção da anestesia foi realizada por via venosa, com infusão de $5 \mathrm{mg} / \mathrm{kg} / \mathrm{h}$ de tiopental;

6. Em seguida, foi feita anestesia local, na face interna de uma das coxas do animal, com $5 \mathrm{ml}$ de lidocaína, $1 \%$ sem vasoconstritor para incisão e cateterização da artéria femoral e medida da pressão arterial contínua. Posteriormente, e pela mesma incisão, foi dissecada a veia femoral e introduzido cateter de Swan-Ganz $7 F$ até um ramo de uma das artérias pulmonares, confirmado pelo aspecto morfológico da curva de pressão obtida. Assim, foi possível medir o débito cardíaco (DC) por termo-diluição, as pressões arteriais médias (PAm) e a freqüência cardíaca (FC). Por meio de fórmula clássica consagrada na literatura, o monitor realizou também o cálculo do índice cardíaco (IC). Neste primeiro tempo, colheu-se uma amostra sangüínea para dosagem de hematócrito e hemoglobina dos animais;

7. Após período de cerca de 30 minutos de estabilização e repouso, foi realizada a primeira série de medidas hemodinâmicas, e estas foram consideras o padrão (MI);

8. Em seguida, os animais foram aleatoriamente, em duplocego, divididos em dois grupos: grupo bupivacaína (B) e grupo mistura enantiomérica $(\mathrm{E})$, e neles foi praticada injeção endovenosa de uma dose tóxica de $4 \mathrm{mg} / \mathrm{kg}$ do anestésico local|' ${ }^{13}$ correspondente, sem o conhecimento do experimentador;

9. Novas medidas hemodinâmicas foram realizadas a um

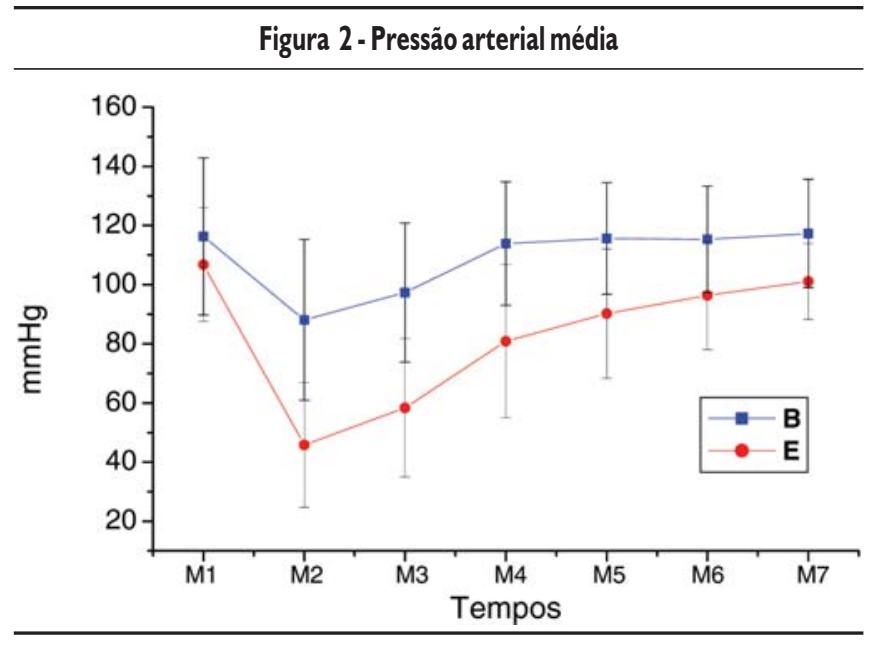

minuto, aos cinco, 10, 15, 20 e 30 minutos após a intoxicação (M2 a $M 7$ respectivamente);

10. Ao término, o animal foi sacrificado, ainda sob o efeito da anestesia, com injeção endovenosa de $10 \mathrm{ml}$ de cloreto de potássio a $19,1 \%$.

Posteriormente, o duplo-cego foi revelado e os dados tratados estatisticamente. As variáveis categóricas receberam tratamento estatístico pelo teste de Qui quadrado. Para comparar a distribuição de variáveis numéricas medidas em momento único, foi utilizado o teste $T$ de Student, e para estudo das variáveis numéricas medidas nos vários tempos, foi utilizada a análise de variância para medidas repetidas (ANOVA). Foram ainda utilizados o teste de Duncan, para comparar os grupos em cada momento, e o teste de perfil de contrastes, para analisar a evolução entre os tempos em cada grupo. O nível de significância adotado foi de 5\%, ou seja, p d" 0,05.

\section{Resultados}

Os grupos foram homogêneos na distribuição por sexo $(p=0,803)$, peso $(p=0,73)$, hematócrito $(p=0,43)$, hemoglobina $(p=0,19)$ e superfície corpórea $(p=0,69)$. Não houve diferença entre os grupos no repouso em nenhum dos parâmetros hemodinâmicos medidos.

Após a intoxicação, houve queda do índice cardíaco (Figura I) nos dois grupos, esta porém foi estatisticamente mais importante no grupo $E$, assim se mantendo até M6 $(p=0,004)$. Até o fim do experimento, em ambos os grupos, o índice cardíaco não retornou a valores semelhantes aos de repouso $(p<0,00 \mathrm{l})$.

Houve queda significativa da FC (Figura 3) após a intoxicação, nos dois grupos, porém esta foi mais importante em $E$, e esta diferença se manteve até o fim do experimento $(p<0,00 I)$. Nos dois grupos, a FC não retornou a valores comparáveis aos de repouso até $M 7(p=0,003)$.

\section{Discussão}

A levobupivacaína proporciona menor bloqueio motor do que a mistura racêmica ${ }^{7,14-17}$, por esta razão, talvez houvesse um interesse na adição de pequena quantidade do isômero dextrógiro para 


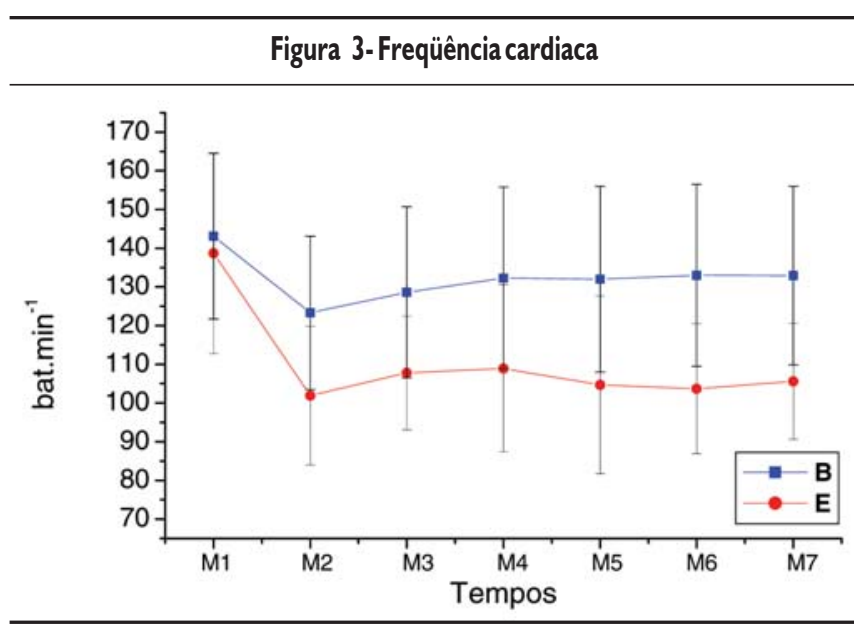

minimizar o efeito ${ }^{16}$. A indústria nacional comercializou este produto e, desde então, ele tem larga utilização em nosso meio, sendo reputado, inclusive, por menor toxicidade.

Tratando-se de formulação existente somente em nosso país, propusemo-nos a investigar sua cardiotoxicidade em suínos, comparando com os efeitos da bupivacaína racêmica. A menor toxicidade cardíaca do isômero S(-) seria devido à sua menor afinidade pelos canais de sódio das células cardíacas, inferior àquela do isômero $R(+)^{18}$ que foi demonstrada em cobaias. Tais dados, no entanto, devem ser olhados com alguma reserva antes de serem extrapolados para humanos.

Em consonância ao demonstrado por outros autores, também em animais, comparando levobupivacaína, bupivacaína racêmica, ropivacaína, e que encontraram toxicidade semelhante ${ }^{19}$ ou mesmo maior $^{20,21}$ da levobupivacaína, nossos resultados mostraram uma maior repercussão hemodinâmica da mistura enantiomérica e, em menor grau, da bupivacaína racêmica num modelo suíno de intoxicação aguda, como a que acontece por ocasião de injeção intravenosa acidental na prática de uma anestesia loco-regional. Tal fato foi evidente na queda significativamente mais importante do índice cardíaco, da pressão arterial média, da freqüência cardíaca e do índice de trabalho sistólico do ventrículo esquerdo.

Estes resultados confirmam, em modelo mais homogêneo, resultados obtidos anteriormente comparando-se estas drogas em cães $^{22}$. Resultados em animais devem ser enxergados com cautela, sua extensão a humanos requer certa prudência, no entanto, devem permitir reflexões e incentivar estudos mais aprofundados, haja vista de tratarem-se de uma segunda série com resultados semeIhantes.

Acidentes por ocasião de anestesias loco-regionais com injeção intravascular de doses elevadas e reações tóxicas, vêm se reduzindo drasticamente nos últimos 30 anos, caindo de 0,2 \% a 0,01\%, e os bloqueios nervosos periféricos ainda respondem pela maioria destes casos (7,5 por 10.000)23. Tais reflexões devem estimular novos esforços, de maneira a chegarmos a drogas e técnicas que permitam alcançar uma morbi-mortalidade significativamente pequena, para proteger nossos pacientes de efeitos indesejáveis e imprevisíveis das técnicas de anestesia loco-regional com altas doses de anestésicos.

\section{ConClusão}

Este trabalho mostrou, em suínos, que a mistura enantiomérica é mais cardiotóxica do que a bupivacaína racêmica, quando grandes doses são injetadas por via endovenosa, como acidentalmente acontece durante anestesias loco-regionais.

\section{Agradecimentos}

Ao Laboratório Cristália

\section{Conflito de interesse: não há.}

\section{SUMMARY}

HAEMODYNAMIC EFFECTS OF INTOXICATION WITH BUPIVACAINE AND ENANTIOMERIC EXCESS MIXTURE. EXPERIMENTAL STUDY IN SWINE

BACKGROUND. Racemic bupivacaine has been the local anaesthetic of choice in regional blocks due to quality and duration of anesthesia. However its cardiovascular toxicity has been a source of concern and research has been made for lesser impact drugs. One choice is its levogyre isomer, levobupivacaine, apparently less cardiotoxic due a lower affinity to the heart sodium channels. In Brazil, a drug containing $75 \%$ of levogyre isomer and $25 \%$ of dextrogyre isomer, called enantiomeric excess mixture, is available. This study intends to evaluate haemodynamic effects of the intravascular injection of a toxic dose of both agents in swine.

Methods. Large White pigs were anaesthetized with thiopental, intubated and placed on mechanical ventilation. Haemodynamic monitoring was performed with an invasive blood pressure and Swan-Ganz catheter on a pulmonary artery. After a 30 minute rest period, animals were randomly divided in two groups and the intoxication was performed on a double-blind method with $4 \mathrm{mg} . \mathrm{kg}$ I of one of the drugs. Haemodynamic parameters were then evaluated at 1, 5, 10, 15, 20 and 30 minutes.

RESULTS. The enantiomeric excess mixture caused greater haemodynamic effects than the racemic bupivacaine. These results diverge from those found in humans with levogyre isomer but are similar to recent results reported in animals. Care should be taken when extrapolating data obtained in swine to humans and further research is necessary.

Conclusion. When high doses are injected in swine, the enantiomeric excess mixture was more toxic than the racemic bupivacaine. [Rev Assoc Med Bras 2007; 53(6): 502-5]

KEY WORDS: Anesthetics. Bupivacaine. Enantiomeric excess mixture. Haemodynamic. Intoxication.

\section{REFERÊNCIAS}

I. Groban L, Deal DD, Vernon JC et al - Cardiac resuscitation after incremental overdosage with lidocaine, bupivacaine, levobupivacaine, and ropivacaine in anesthetized dogs. Anesth Analg 2001;92:37-43.

2. Chang DH-T, Ladd LA, Copeland S et al - Direct cardiac effects of intracoronary bupivacaine, levobupivaciane and ropivacaine in the 
sheep. Br J Pharmacol 2001;132:649-658.

3. Albright GA - Cardiac arrest following regional anesthesia with etidocaine or bupivacaine. Anesthesiology 1979;51:285-287.

4. Ohmura S, Kawada M, Ohta T et al - Systemic toxicity and resusciation in bupivacaine, levobupivacaine, or ropivacaine-infused rats. Anesth Analg 2001:93:743-748.

5. Aberg G - Toxicological and local anaesthetic effects of optically active isomers of two local anaesthetic compounds. Acta Pharmacol Toxicol 1972;31:273-286.

6. Luduena FP, Bogado EF, Tullar BF - Optical isomers of mepivacaine and bupivacaine. Arch Int Pharmacodyn 1972;200:359-369.

7. Foster RH, Markham A - Levobupivacaine. A review of its pharmacology and use as a local anaesthetic. Drugs 2000;59:55 I-579.

8. Lyons G, Columb M, Wilson RC et al. - Extradural pain relief in labour:potencies of levobupivacaine and racemic bupivacaine. $\mathrm{Br}$ J Anaesth, 1998;81:899-901.

9. Héctor JL, Columb MO - The relative motor blocking potencies of bupivacaine and levobupivacaine in labour. Anesth Analg, 2003;97: I 509-1513.

10. Bardsley H, Gristwood R, Baker $H$ et al - A comparison of the cardiovascular effects of levobupivacaine and rac-bupivacaine following intravenous administration to healthy volunteers. Br Clin Pharmacol. 1 998:46:245-9.

I I. Smith AC, Ehler WJ, SwindleMMI. Anesthesia and analgesia in swine, em: Kohn DF, Wixson SK, White WJ, et al. Anesthesia and analgesia in laboratory animals. New York: Academic Press; 1997. p.313-36.

12. Ettinger SJ. Textbook of veterinary internal medicine. Philadelphia: WB Saunders; 1975. p.| 146.

13. Lefrant JY, Muller L, La Coussaye JE, Lalourcey L, et al. Hemodynamic and cardiac electrophysiologic effects of lidocaine-bupivacaine mixture in anesthetized and ventilated piglets. Anesthesiology. 2003;98:96103.

14. Delfino J, Vale NB. Bupivacaína levógira a 0,5\% pura versus mistura enantiomérica de bupivacaína (S75-R25) a 0,5\% em anestesia peridural para cirurgia de varizes. Rev Bras Anestesiol. 2001 ; 51:474-81.
I5. Tanaka PP, Souza RO, Salvalaggio MFO, Tanaka MAA. Estudo comparativo entre bupivacaína a $0,5 \%$ e a mistura enantiômera de bupivacaína (S75-R25) a 05\% em anestesia peridural em pacientes submetidos a cirurgia ortopédica de membros inferiores. Rev Bras Anestesiol. 2003;53: 33I-337.

16. Gonçalves RF, Lauretti GR, Mattos Al. Estudo comparativo entre bupivacaína 0,5\% e mistura enantiomérica de bupivacaína (S75-R25) em anestesia peridural. Rev Bras Anestesiol. 2003;53:169-76.

17. Lacassie HJ, Columb MO. The relative motor blocking potencies of bupivacaine and levobupivacaine in labor. Anesth Analg. 2003;97:1509-13.

18. Valenzuela C, Snyders DJ, Bennett PB, et al. Stereosectivite block of cardiac sodium channels by bupivacaine in guinea pig ventricular myocytes. Circulation. 1995;92:30|4-24.

19. Royse CF, Royse AG. The myocardial and vascular effects of bupivacaine, levobupivacaine, and ropivacaine using pressure volume loops. Anesth Analg. 2005; 101:679-87.

20. Masuda R, Takeda S, Yoshii S, et al. Levobupivacaine exerts the most detrimental effect on the cardiovascular system among enantiomers of bupivacaína in anesthetized dogs. Anesthesiology. 2004;101:A652.

21. Jung CW, Lee $\mathrm{KH}$, Choe YS, et al. Comparison of resuscitative effect of insulin between bupivacaine and levobupivacaína induced cardiovascular collapse in dogs. Anesthesiology. 2004;101:A649.

22. Udelsmann A, Munhoz DC, Silva WA, Moraes AC, Marcondes G. Comparação entre os efeitos hemodinâmicos da intoxicação aguda com bupivacaína racêmica e a mistura com excesso enatiomérico de 50\% (S75-R25). Estudo experimental em cães. Rev Bras Anestesiol. 2006;56:39I-40I.

23. Cox B, Durieux ME, Marcus MA. Toxicity of local anaesthetics. Best Pract Res Clin Anaesthesiol. 2003;17:1 |1-36.

Artigo recebido: 12/02/07

Aceito para publicação: 15/09/07 